\title{
Optical Properties of Plasmas Based on an Average-Atom Model
}

\author{
W. R. Johnson \\ Dept. of Physics, 225 Nieuwland Science Hall \\ University of Notre Dame, Notre Dame, IN 46556 \\ C. Guet \\ CEA/DAM Ile de France \\ BP12, 91680 Bruyères-le-Châtel, France \\ G. F. Bertsch \\ Dept. of Physics and Inst. Nuclear Theory \\ Box 351560, University of Washington, Seattle, WA 98915
}

\begin{abstract}
Optical properties of plasmas, including dielectric constants, indices of refraction, and absorption coefficients, are determined from an average-atom point of view. Linear response of an average atom to an harmonic electric field leads to an averageatom version of the Kubo-Greenwood formula, which is used to calculate the frequencydependent electric conductivity of the plasma. The frequency-dependent dielectric function is determined from the conductivity using Kramers-Kronig dispersion relations. The index of refraction and absorption coefficient of the plasma are subsequently obtained from the dielectric function. Comparing the present results with the free-electron model helps one understand anomalies observed recently in space and time resolved interferograms of $\mathrm{Al}$ plasmas produced by $13.9 \mathrm{~nm}$ and $14.7 \mathrm{~nm}$ x-ray lasers.
\end{abstract}

Key words: plasma, conductivity, dielectric function, index of refraction, dispersion relations, x-ray interferometry

PACS: 51.70.+f, 52.25.Mq, 32.70.Cs, 32.80.Fb

Preprint submitted to Elsevier Science

17 November 2004 


\section{Introduction}

X-ray interferograms are widely used in plasma diagnostics owing to the fact that they can be interpreted in terms of the index of refraction $n$, from which the electron density can be determined. The free-electron model, which is frequently used to interpret the index of refraction, predicts that $n<1$. Recently, however, instances where $n>1$ have been observed in interferograms of an Al plasma produced by a $14.7 \mathrm{~nm}$ Ni-like Pd soft x-ray laser at the Lawrence Livermore National Laboratory COMET laser facility [1]. Similar anomalies were noted in interference patterns of an Al plasma produced by a $13.9 \mathrm{~nm}$ Ni-like Ag laser at the Advanced Photon Research Center of the Japan Atomic Energy Research Institute [2]. These anomalies have been attributed to the influence of bound electrons on the optical properties of the plasma [1-3].

In the present work, we study optical properties of plasmas theoretically starting from an average-atom cell-model and confirm that at low temperatures (1-4 eV) the index of refraction of a dense $\mathrm{Al}$ plasma is indeed greater than 1 for photon wavelengths in the range 10-15 $\mathrm{nm}$. Our average-atom model is a quantum mechanical version of the generalized Thomas-Fermi model of a plasma devised more than a half-century ago by Feynman, Metropolis, and Teller [4] and is similar to a model used previously by Blenski and Ishikawa [5]. Applying linear response theory to the average atom leads to a version of the Kubo-Greenwood ( $\mathrm{KG}$ ) formula [6-10] for the the frequency-dependent conductivity $\sigma(\omega)$ of the plasma. It should be noted that the KG formula has also been used recently in Refs. [11,12] to study plasma conductivity from a molecular dynamics point of view. Conductivity is related to the imaginary part of the relative dielectric function $\epsilon_{r}(\omega)$; therefore, with the aid of Kramers-Kronig dispersion relations [13-16], one can determine the entire complex dielectric function $\epsilon_{r}(\omega)$ and the corresponding complex index of refraction $n(\omega)+i \kappa(\omega)=\sqrt{\epsilon_{r}(\omega)}$.

In the following sections, we outline the average-atom model, derive the KuboGreenwood formula, and use a dispersion relation to obtain the dielectric function. Finally, we apply the model to evaluate the index of refraction and absorption coefficient of an aluminum plasma (density $10^{20}$ ions/cc) in a temperature range $T=1-30 \mathrm{eV}$. These results are compared with the free electron model in an effort to explain the anomalies discussed above.

\section{Quantum Mechanical Average-Atom Model}

The plasma is divided into neutral cells, each containing $Z$ electrons, centered on a nucleus of charge $Z$. The radius of each cell is taken to be the Wigner-Seitz 
(WS) radius, determined from the density and atomic weight. Each electron is assumed to satisfy the self-consistent central-field Schrödinger equation

$$
\left[\frac{p^{2}}{2 m}-\frac{Z}{r}+V\right] u_{a}(\boldsymbol{r})=\epsilon u_{a}(\boldsymbol{r})
$$

where $a=(n, l)$ for bound states or $(\epsilon, l)$ for continuum states. The potential inside a cell consists of two parts: $V(r)=V_{\text {dir }}+V_{\text {exc }}$. Outside the cell $V=0$. The direct part of the potential is obtained from

$$
\nabla^{2} V_{\text {dir }}=-4 \pi \rho
$$

where the electron density $\rho=\rho_{b}+\rho_{c}$ has contributions from both bound and continuum electrons. In the present work, the exchange potential is given by the local density approximation

$$
V_{\mathrm{exc}}=-\left(\frac{3}{\pi} \rho(r)\right)^{1 / 3}
$$

The exchange potential could, of course, be replaced by a more realistic exchange-correlation potential such as that given in [17] or [18].

The bound-state contribution to the density is

$$
4 \pi r^{2} \rho_{b}(r)=\sum_{n l} 2(2 l+1) f_{n l} P_{n l}(r)^{2}
$$

where $P_{n l}(r)$ is the bound-state radial wave function,

$$
f_{n l}=\frac{1}{1+\exp \left[\left(\epsilon_{n l}-\mu\right) / k T\right]}
$$

is the Fermi distribution function for a state with quantum numbers $(n l), \mu$ is the chemical potential, and $T$ is the temperature. The summation in Eq. (4) ranges over all subshells. Contributions to the density from continuum electrons $\rho_{c}$ are given by a similar formula with the bound state radial function $P_{n l}(r)$ replaced by the continuum wave function $P_{\epsilon l}(r)$ normalized on the energy scale and the sum over $n$ replaced by an integral over $\epsilon$. To accelerate convergence of the sum over $l$ in the continuum case, we follow the suggestion in Ref. [5] and subtract the free-particle contribution from each partial wave, then add back the exactly known free-particle density.

Finally, the chemical potential $\mu$ is chosen to insure electric neutrality of the Wigner-Seitz cell:

$$
Z=\int_{r<R} \rho(r) d^{3} r \equiv \int_{0}^{R} 4 \pi r^{2} \rho(r) d r
$$

Equations (1-5) above are solved self-consistently to give the chemical potential $\mu$, the electrostatic potential $V(r)$ and the electron density $\rho(r)$. 
Table 1

Average atom of aluminum at density $0.27 \mathrm{gm} / \mathrm{cc}$ and temperature $T=5 \mathrm{eV}$. The Wigner-Seitz radius is $R=6.44$ a.u. and the chemical potential is $\mu=-0.3823$ a.u.. Five bound states are found. The number of electrons contained within the WignerSeitz cell $n(l)$ are also listed.

\begin{tabular}{ccccccc}
\hline \hline \multicolumn{3}{c}{ Bound States } & \multicolumn{5}{c}{ Continuum States } \\
State & Energy & $n(l)$ & $l$ & $n(l)$ & $n_{0}(l)$ & $\Delta n(l)$ \\
\hline $1 s$ & -55.189 & 2.0000 & 0 & 0.1090 & 0.1975 & -0.0885 \\
$2 s$ & -3.980 & 2.0000 & 1 & 0.2149 & 0.3513 & -0.1364 \\
$3 s$ & -0.259 & 0.6759 & 2 & 0.6031 & 0.3192 & 0.2839 \\
$2 p$ & -2.610 & 6.0000 & 3 & 0.2892 & 0.2232 & 0.0660 \\
$3 p$ & -0.054 & 0.8300 & 4 & 0.1514 & 0.1313 & 0.0201 \\
& & & 5 & 0.0735 & 0.0674 & 0.0061 \\
& & & 6 & 0.0326 & 0.0308 & 0.0018 \\
& & & 7 & 0.0132 & 0.0127 & 0.0005 \\
& & & 8 & 0.0049 & 0.0048 & 0.0001 \\
& & & 9 & 0.0017 & 0.0016 & 0.0001 \\
\hline \hline Nbound & & 11.5059 & Nfree & 1.4941 & 1.3404 & 0.1537 \\
\hline \hline
\end{tabular}

\subsection{Example: $A$ l at $T=5 \mathrm{eV}$ and density $0.27 \mathrm{gm} / \mathrm{cc}$}

As an illustration of the present average-atom model, consider aluminum at $0.1 \times$ metallic density and $T=5 \mathrm{eV}$. The corresponding WS radius is $R=$ 6.44 a.u.. The average-atom potential in this case supports five bound states; their energies and the associated number of electrons $n(l)$ inside the WignerSeitz cell are given in Table 1. For each continuum partial wave, we list $n(l)$, $n_{0}(l)$ the number of "free" waves within the cell, and differences $\Delta n(l)=$ $n(l)-n_{0}(l)$, which are seen to converge more rapidly at large $l$. Note that $N_{\text {bound }}+N_{\text {free }}=13$ for overall charge neutrality.

In the left panel of Fig. 1, we show bound and continuum contributions to the radial density for the case described in Table 1 and in the right panel, the continuum contribution to the electron density $\rho_{c}(r)$ is compared with the free electron background density $\rho_{0}$. The density merges smoothly into the background outside the Wigner-Seitz sphere. 

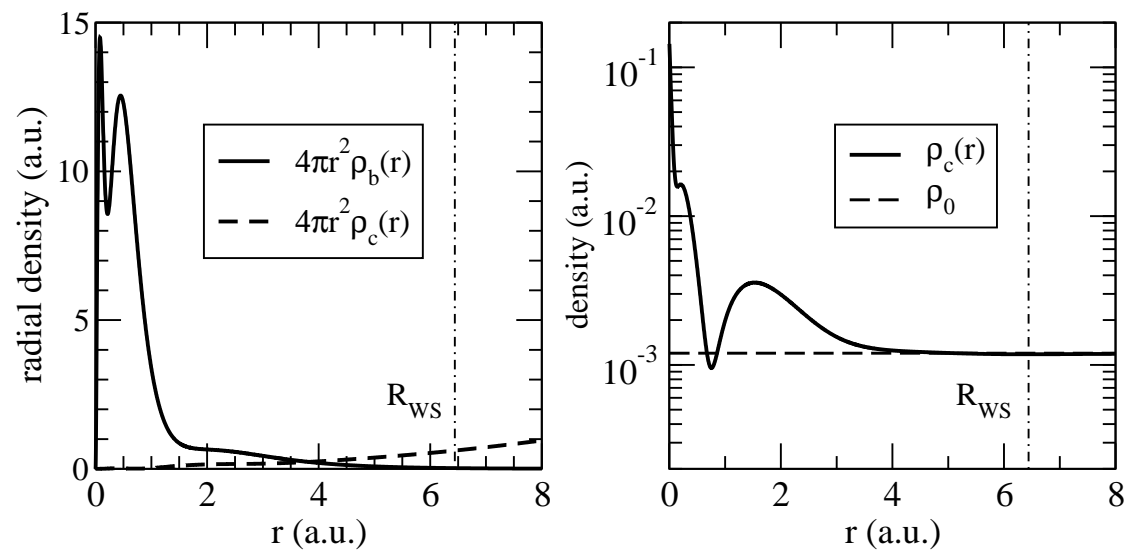

Fig. 1. Left panel: Bound and continuum contributions to the radial electron densities for $\mathrm{Al}$ at $T=5 \mathrm{eV}$ and density $0.27 \mathrm{gm} / \mathrm{cc}$. Right panel: Continuum contribution to the electron density $\rho_{c}(r)$ merges smoothly into the background density $\rho_{0}$.

\section{Linear Response and the Kubo-Greenwood Formula}

We assume that a time dependent electric field is applied to an average atom. We describe the field by the vector potential

$$
\boldsymbol{A}(t)=\frac{F}{\omega} \hat{\boldsymbol{z}} \cos \omega t
$$

The one-electron Hamiltonian is

$$
H=\frac{1}{2 m}\left[p_{x}^{2}+p_{y}^{2}+\left(p_{z}-e A_{z}(t)\right)^{2}\right]+V(n, r)=T+V(n, r)-\frac{e F}{\omega} v_{z} \cos \omega t,
$$

where we have retained only those terms linear in the field strength $F$. The time dependent Schrödinger equation becomes

$$
\left[T_{0}+V(n, r)-\frac{e F}{\omega} v_{z} \cos \omega t\right] \psi_{i}(\boldsymbol{r}, t)=i \frac{\partial}{\partial t} \psi_{i}(\boldsymbol{r}, t) .
$$

We seek a solution to Eq. (7) of the form

$$
\psi_{i}(\boldsymbol{r}, t)=u_{i}(\boldsymbol{r}) e^{-i \epsilon_{i} t}+w_{i}^{+}(\boldsymbol{r}) e^{-i\left(\epsilon_{i}+\omega\right) t}+w_{i}^{-}(\boldsymbol{r}) e^{-i\left(\epsilon_{i}-\omega\right) t},
$$

where $u_{i}(\boldsymbol{r})$ is a solution to the time-independent Schrödinger equation and where $w_{i}^{ \pm}(\boldsymbol{r})$ are small perturbations. We find

$$
\begin{aligned}
{\left[T_{0}+V(n, r)\right] u_{i}(\boldsymbol{r}) } & =\epsilon_{i} u_{i}(\boldsymbol{r}) \\
{\left[T_{0}+V(n, r)-\left(\epsilon_{i} \pm \omega\right)\right] w_{i}^{ \pm}(\boldsymbol{r}) } & =\frac{e F}{2 \omega} v_{z} u_{i}(\boldsymbol{r}) .
\end{aligned}
$$

In writing these equations, we have ignored the modifications of the potential $V(n, r)$ induced by the field. Taking these into account would lead to an average-atom version of the random-phase approximation [19]. 
Next, we carry out an eigenvalue expansion of the perturbed orbital as

$$
w_{i}^{+}(\boldsymbol{r})=\sum_{j} X_{i}^{j} u_{j}(\boldsymbol{r}), \quad w_{i}^{-}(\boldsymbol{r})=\sum_{j} Y_{i}^{j} u_{j}(\boldsymbol{r})
$$

From (9), we find

$$
X_{i}^{j}=\frac{e F}{2 \omega} \frac{\left\langle j\left|v_{z}\right| i\right\rangle}{\epsilon_{j}-i \eta-\epsilon_{i}-\omega}, \quad Y_{i}^{j}=\frac{e F}{2 \omega} \frac{\left\langle j\left|v_{z}\right| i\right\rangle}{\epsilon_{j}-i \eta-\epsilon_{i}+\omega} .
$$

The current associated with the perturbed atom is

$$
\begin{aligned}
J_{z}(t)=\frac{2 e}{\Omega} \sum_{i} f_{i}\left\langle\psi_{i}(t)\left|v_{z}\right| \psi_{i}(t)\right\rangle & \\
& =\frac{2 e}{\Omega} \sum_{i} f_{i}\left[\left(\left\langle u_{i}\left|v_{z}\right| w_{i}^{+}\right\rangle+\left\langle w_{i}^{-}\left|v_{z}\right| u_{i}\right\rangle\right) e^{-i \omega t}+\text { c.c. }\right]
\end{aligned}
$$

to terms linear in $F$. In the above equation $\Omega$ is the volume/atom. The Fermi distribution function $f_{i}$ for state $i$ accounts for the initial state occupation in the average atom and the factor of 2 in Eq. (10) accounts for two spin states/electron.

The response current may be rewritten in terms of the expansion coefficients as

$$
J=\frac{2 e}{\Omega} \sum_{i j} f_{i}\left[\left(\left\langle i\left|v_{z}\right| j\right\rangle X_{i}^{j}+\left\langle j\left|v_{z}\right| i\right\rangle Y_{i}^{j \star}\right) e^{-i \omega t}+\text { c.c. }\right]
$$

The conductivity is determined by the part of the current $J_{1}$ in phase with the driving field:

$$
J_{1}=\frac{4 e}{\Omega} \sum_{i j} f_{i} \Im\left(\left\langle i\left|v_{z}\right| j\right\rangle X_{i}^{j}+\left\langle j\left|v_{z}\right| i\right\rangle Y_{i}^{j \star}\right) \sin \omega t
$$

One finds:

$$
\begin{aligned}
& \Im\left(\left\langle i\left|v_{z}\right| j\right\rangle X_{i}^{j}+\left\langle j\left|v_{z}\right| i\right\rangle Y_{i}^{j \star}\right) \\
& \quad=\frac{e F}{2 \omega} \sum_{j}\left(\pi \delta\left(\epsilon_{j}-\epsilon_{i}-\omega\right)\left|\left\langle i\left|v_{z}\right| j\right\rangle\right|^{2}-\pi \delta\left(\epsilon_{j}-\epsilon_{i}+\omega\right)\left|\left\langle j\left|v_{z}\right| i\right\rangle\right|^{2}\right) .
\end{aligned}
$$

Interchanging indices $i \leftrightarrow j$ in second term leads to $J_{1}(t)=\sigma(\omega) E_{z}(t)$ with

$$
\sigma(\omega)=\frac{2 \pi e^{2}}{\omega \Omega} \sum_{i j}\left(f_{i}-f_{j}\right)\left|\left\langle j\left|v_{z}\right| i\right\rangle\right|^{2} \delta\left(\epsilon_{j}-\epsilon_{i}-\omega\right),
$$

which is an average-atom version of the Kubo-Greenwood formula. 


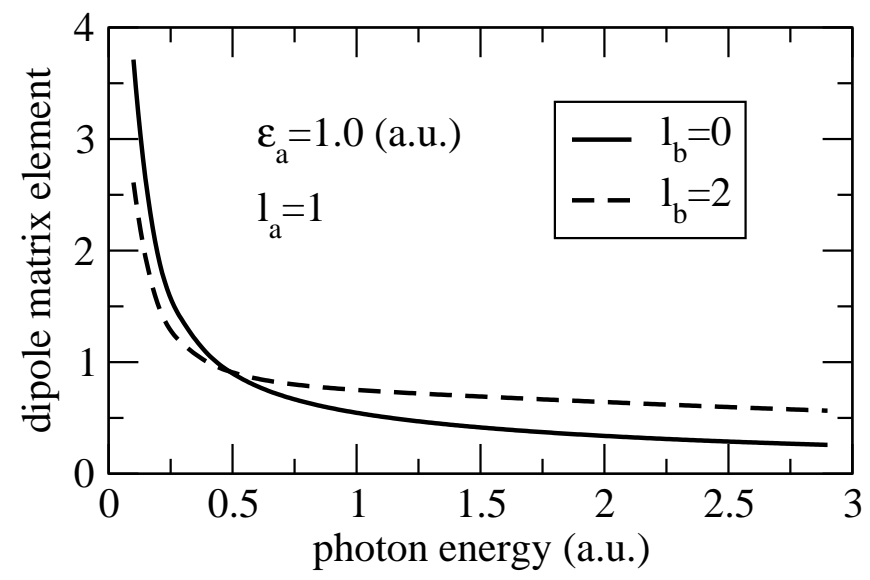

Fig. 2. Test radial matrix elements $\left\langle\epsilon_{a} l_{a}\|v\| \epsilon_{a}+\omega l_{b}\right\rangle$ for an average atom of $\mathrm{Al}(5 \mathrm{eV}$ and 0.1 metallic density) are given as functions $\omega$. The initial energy $\epsilon_{a}$ is 1 a.u. and the initial angular momentum $l_{a}=1$.

\subsection{Reduction of the Kubo-Greenwood Formula}

There are three distinct contributions to Eq. (13) arising from free-free transitions, bound-bound transitions (line spectrum) and from bound-free transitions (photoabsorption).

\subsubsection{Free-Free:}

The free-free (inverse bremsstrahlung contribution) to the conductivity which dominates near $\omega=0$ where other channels are closed is given by

$$
\sigma(\omega)=\frac{2 \pi e^{2}}{3 \omega \Omega} \sum_{l_{i}} \int_{0}^{\infty} d \epsilon_{i}\left(f_{i}-f_{j}\right)\left[\left|\left\langle\epsilon_{i} l_{i}\|v\| \epsilon_{j} l_{i}+1\right\rangle\right|^{2}+\left|\left\langle\epsilon_{i} l_{i}\|v\| \epsilon_{j} l_{i}-1\right\rangle\right|^{2}\right]
$$

with $\epsilon_{j}=\epsilon_{i}+\omega$.

Sample matrix elements for a test case with $E_{i}=1$ a.u. and $l_{i}=2$ are shown in Fig. 2 as functions of $\omega$, where $\omega=E_{f}-E_{i}$. It is apparent from the figure that the radial matrix elements diverge as $\omega^{-1}$ for small $\omega$. This behavior is a consequence of the fact that we have ignored interactions of electrons with ions outside the WS cell in our average-atom model.

Owing to the infrared divergence in radial matrix elements, the conductivity diverges as $1 / \omega^{2}$. This low-frequency divergence would be protected in more sophisticated calculations by finite lifetimes of excited states. In the present calculation, we mock up such contributions using a scaling factor $\omega^{2} /\left(\gamma^{2}+\omega^{2}\right)$ in the integrand of the $\mathrm{KG}$ formula to remove the divergence. The resulting 
conductivity behaves as

$$
\sigma(\omega) \approx \frac{\sigma(0) \gamma^{2}}{\gamma^{2}+\omega^{2}}
$$

for small $\omega$, in harmony with the classical Drude model $[20,21]$ of conductivity. In the classical model of conductivity, $\gamma$ is the mean electron-ion collision rate. In the present work, the scaling factor $\gamma$ is chosen in such a way that the freefree contribution to the conductivity sum rule:

$$
\int_{0}^{\infty} \sigma(\omega) d \omega=\frac{\pi}{2} \frac{e^{2} N_{\text {free }}}{m \Omega}
$$

is satisfied. Here $N_{\text {free }}$ is the number of free electrons/ion and $\Omega$ is the WignerSeitz cell volume or, equivalently, the volume/ion.

For the example of $\mathrm{Al}$ at $T=5 \mathrm{eV}$ and density $0.27 \mathrm{gm} / \mathrm{cc}$ discussed in the previous section, the number of free electrons/ion is 1.494 leading to $\gamma=$ 0.0466 a.u. $=1.27 \mathrm{eV}$. The limiting value of the conductivity in this case is $\sigma(0)=0.0262$ a.u. $=0.121(\mu \Omega-\mathrm{m})^{-1}$. It should be noted that scaling has no effect for $\omega \gg \gamma$. Results of evaluating Eq. (14) with scaling are shown in the lower left panel of Fig. 3. The static conductivity obtained from the Ziman formula [22, chap. 8] for the example of $\mathrm{Al}$ at $T=5 \mathrm{eV}$ and density $0.27 \mathrm{gm} / \mathrm{cc}$ is $\sigma_{\text {Ziman }}=0.125(\mu \Omega-\mathrm{m})^{-1}$ in good agreement with the present value.

\subsubsection{Bound-Bound:}

The contribution to the conductivity from bound-bound transitions is

$$
\sigma(\omega)=\frac{2 \pi e^{2}}{3 \Omega} \sum_{n_{i} l_{i} n_{j} l_{j}} \frac{\left(f_{i}-f_{j}\right)}{\omega_{i j}}\left|\left\langle n_{i} l_{i}\|v\| n_{j} l_{j}\right\rangle\right|^{2},
$$

where $\omega_{i j}=\epsilon_{j}-\epsilon_{i}>0$. This leads to a finite number of discrete line contributions.

To give a specific example, we return to the case of $\mathrm{Al}$ at density $0.27 \mathrm{gm} / \mathrm{cc}$ and $T=5 \mathrm{eV}$. Four transitions listed in Table 2 contribute to the conductivity. Two other possibilities permitted by dipole selection rules $(1 s \rightarrow 2 p$ and $2 s \rightarrow$ $2 p$ ) do not contribute since $n=1$ and $n=2$ states are completely occupied in this example. Three of the four bound-bound transitions are also illustrated in the upper left panel of Fig. 3, where we have folded the lines into a Lorentzian line profile of width $\gamma$ given by the effective collision rate discussed in the previous subsection. The effective number of electrons contributing to the bound-bound part of the conductivity sum rule in this example is $N_{b b}=0.493$. 
$\mathrm{Al} \mathrm{T}=5 \mathrm{eV}$ density $=0.27 \mathrm{gm} / \mathrm{cc}$
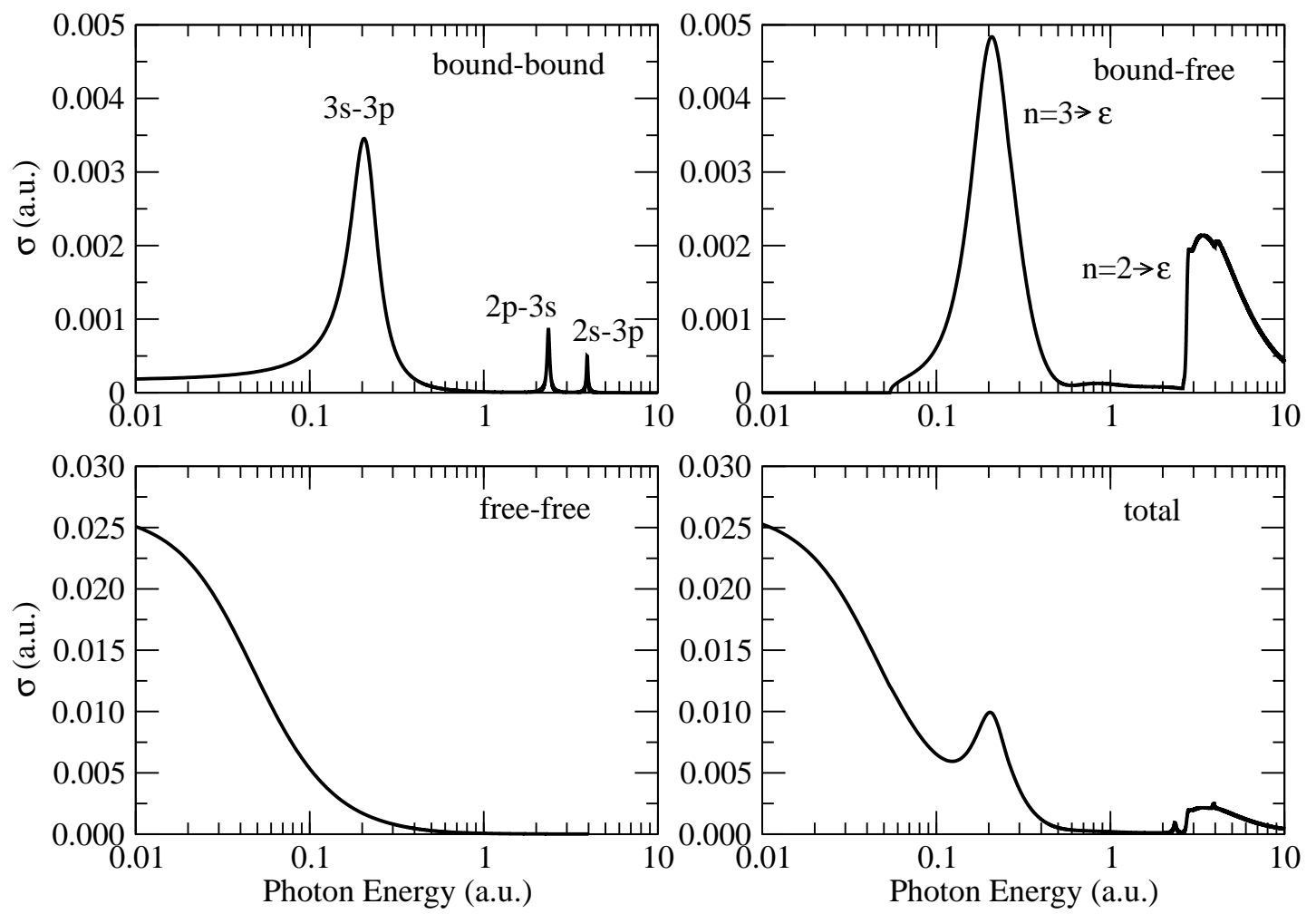

Fig. 3. Contributions to the conductivity for an average atom of $\mathrm{Al}$ (5eV and 1/10 metallic density) is given as functions of the photon energy. Upper left: bound-bound. Upper right: bound-free. Lower left: free-free. Lower right: total $\sigma(\omega)$.

Table 2

Bound-bound contributions to the conductivity for $\mathrm{Al}$ at $\mathrm{T}=5 \mathrm{eV}$ and density 0.27 $\mathrm{gm} / \mathrm{cc}$.

\begin{tabular}{ccc}
\hline \hline$i \rightarrow j$ & $\omega_{i j}$ & $\sigma\left(\omega_{i j}\right)$ \\
\hline $3 s \rightarrow 3 p$ & 0.205 & 0.0004702 \\
$2 p \rightarrow 3 s$ & 2.350 & 0.0001248 \\
$2 s \rightarrow 3 p$ & 3.926 & 0.0000726 \\
$1 s \rightarrow 3 p$ & 55.135 & 0.0000239 \\
\hline Sum & & 0.0006914 \\
\hline \hline
\end{tabular}

\subsubsection{Bound-Free:}

The contribution to the Kubo formula from bound-free (photoionization) channels is

$$
\sigma(\omega)=\frac{2 \pi e^{2}}{3 \omega \Omega} \sum_{n_{i} l_{i}}\left(f_{i}-f_{j}\right)\left[\left|\left\langle n_{i} l_{i}\|v\| \epsilon_{j} l_{i}+1\right\rangle\right|^{2}+\left|\left\langle n_{i} l_{i}\|v\| \epsilon_{j} l_{i}-1\right\rangle\right|^{2}\right]
$$


where $\epsilon_{j}=\epsilon_{i}+\omega$. For each occupied subshell $n_{i} l_{i}$ there are contributions for all $\omega>-\epsilon_{i}$.

In Fig. 3 we show the contribution to the conductivity given by Eq. (17) for our example of an average atom at density $0.27 \mathrm{gm} / \mathrm{cc}$ and $T=5 \mathrm{eV}$. The prominent low-energy peak is associated with ionization of the partially occupied $3 p$ and $3 s$ subshells. The irregular shoulder near 3 a.u. is caused by opening of the $2 p$ and $2 s$ ionization channels. The contribution to the conductivity from the $1 \mathrm{~s}$ shell, which is ionized at 55 a.u., is not shown in the figure. The sum of the three contributions discussed above is shown in the lower right panel of Fig. 3.

\section{Dispersion Relations}

The complex dielectric function is related to the complex conductivity through the relation

$$
\epsilon_{r}(\omega)=1+i \frac{4 \pi \sigma(\omega)}{\omega}
$$

Thus the imaginary part of the dielectric function is defined once the real part of the conductivity is given by the $\mathrm{KG}$ formula,

$$
\Im \epsilon_{r}(\omega)=\frac{4 \pi}{\omega} \Re \sigma(\omega)
$$

On the general grounds of causality, one may infer the real part of the dielectric function from its imaginary part using the Kramers-Kronig dispersion relations [13-16]. In turn Eq. (18) provides the imaginary part of the conductivity. It follows that the real and imaginary parts of the complex conductivity also satisfy a dispersion relation,

$$
\Im \sigma\left(\omega_{0}\right)=-\frac{2 \omega_{0}}{\pi} f_{0}^{\infty} \frac{\Re \sigma(\omega)}{\omega^{2}-\omega_{0}^{2}} d \omega
$$

In the high frequency limit, the dispersion relation given in Eq. (20) takes the form

$$
\Im \sigma\left(\omega_{0}\right) \rightarrow \frac{2}{\pi} \frac{1}{\omega_{0}} \int_{0}^{\infty} \Re \sigma(\omega) d \omega .
$$

In virtue of the conductivity sum rule used earlier, Eq. (21) shows that $\Im \sigma(\omega)$ has the limiting value $n_{e} e^{2} /\left(m \omega_{0}\right)$, in harmony with the free electron limit of the dielectric function

$$
\epsilon_{r} \rightarrow 1-\frac{4 \pi n_{e} e^{2}}{m \omega^{2}}
$$

Let us apply the dispersion relation (20) to the example of $\mathrm{Al}$ at density 0.27 $\mathrm{gm} / \mathrm{cc}$ and $T=5 \mathrm{eV}$ discussed in the previous section. In Fig. 4, we show 


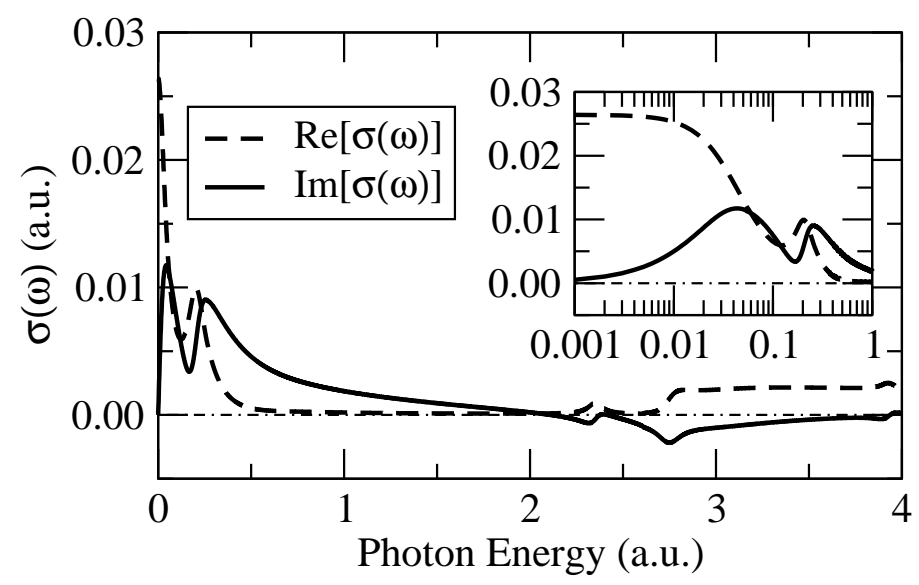

Fig. 4. Real part of $\sigma(\omega)$ (dashed lines) and imaginary parts of $\sigma(\omega)$ (solid lines) for $\mathrm{Al}$ at $5 \mathrm{eV}$ and density 0.27 a.u..

$\Re \sigma(\omega)$ from the earlier KG analysis as dashed curve and we show $\Im \sigma(\omega)$ from the dispersion relation (20) as solid curves.

With the aid of Eq. (18), we can reconstruct the complex dielectric function in terms of these two functions and completely describe the optical properties of the plasma.

\subsection{Index of Refraction}

The real and imaginary parts of the relative dielectric function $\epsilon(\omega)$ are given by Eqs. (18), which in atomic units reduce to

$$
\Re \epsilon_{r}(\omega)=1-4 \pi \frac{\Im \sigma(\omega)}{\omega} \quad \Im \epsilon_{r}(\omega)=4 \pi \frac{\Re \sigma(\omega)}{\omega},
$$

where we have made use of the fact that $1 / \epsilon_{0}=4 \pi$ a.u.. The complex index of refraction $n+i \kappa$ is related to $\epsilon(\omega)$ through the equation

$$
n+i \kappa=\sqrt{\epsilon_{r}}
$$

It follows that

$$
\begin{aligned}
& n(\omega)=\sqrt{\frac{\sqrt{\left[\Re \epsilon_{r}(\omega)\right]^{2}+\left[\Im \epsilon_{r}(\omega)\right]^{2}}+\Re \epsilon_{r}(\omega)}{2}} \\
& \kappa(\omega)=\sqrt{\frac{\sqrt{\left[\Re \epsilon_{r}(\omega)\right]^{2}+\left[\Im \epsilon_{r}(\omega)\right]^{2}}-\Re \epsilon_{r}(\omega)}{2}} .
\end{aligned}
$$

The two functions $n(\omega)$ and $\kappa(\omega)$ are illustrated in Fig. 5, where they are plotted for the example of $\mathrm{Al}$ at $T=5 \mathrm{eV}$ and density $0.27 \mathrm{gm} / \mathrm{cc}$. 


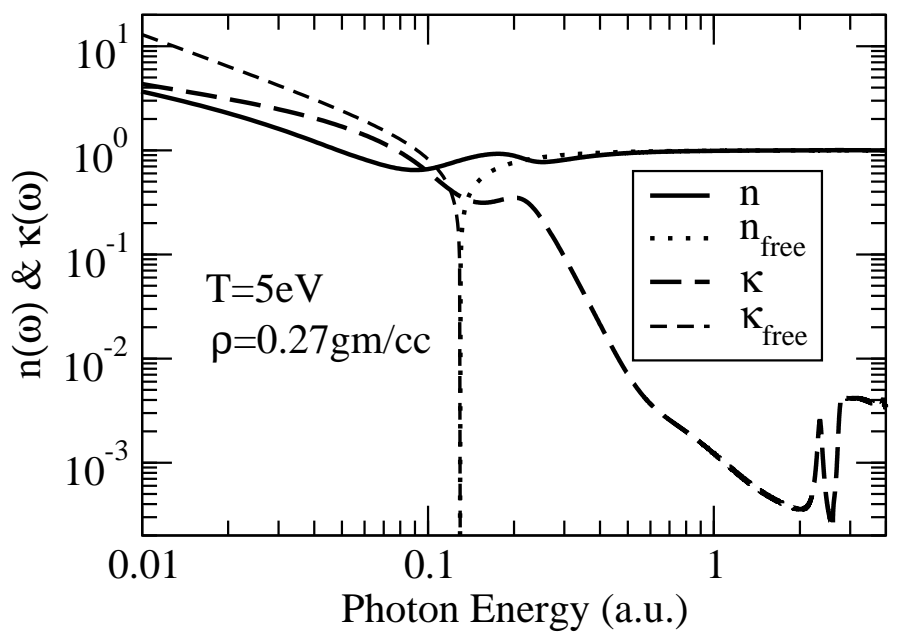

Fig. 5. Real $n(\omega)$ and imaginary $\kappa(\omega)$ parts of the index of refraction for an average atom of $\mathrm{Al}(5 \mathrm{eV}$ and 1/10 metallic density) given as functions of the photon energy $\omega$. The lightly dashed and dotted lines give $n$ and $\kappa$ in the free-electron approximation. The singularity in the free-electron case occurs at the plasma frequency.

\section{$5 \quad$ Application to an Al plasma}

As mentioned in the introduction, the analysis of interferograms produced by $13.9 \mathrm{~nm}$ and $14.7 \mathrm{~nm}$ x-ray lasers gave a plasma index of refraction $n>1$ in some instances. When analyzed in terms of the free-electron model this leads to the conclusion that the square of the plasma frequency $\omega_{0}^{2}$, which is proportional to number of free electrons per ion, is negative in the few $\mathrm{eV}$ temperature range! In [2] this behavior was attributed to the $2 p-3 d$ transition in $\mathrm{Al}^{+2}$. To examine this anomalous behavior in terms of the average-atom model, we compare $n(\omega)-1$ predicted by the average-atom model with its free electron counterpart $n_{\text {free }}(\omega)-1 \approx-\omega_{0}^{2} /\left(2 \omega^{2}\right)$. In the lower panel of Fig. 6 , we plot the ratio $(n-1) /\left(n_{\text {free }}-1\right)$ over the range of photon energies $\omega=0$ to $100 \mathrm{eV}$ for temperature $T=3 \mathrm{eV}$. The number of free electrons per ion from the average-atom model is $\langle Z\rangle=1.38$ for this density and temperature. As can be seen in the figure, the ratio is near -2 in a band of photon energies that include those of the $\mathrm{Pd}$ and $\mathrm{Ag}$ lasers indicated by the dashed lines at 84 and $89 \mathrm{eV}$. This explains the behavior seen experimentally; the main effect on the dispersion integral in this region comes from $2 \mathrm{p}-2 \mathrm{~s}$ and $2 \mathrm{p}-3 \mathrm{~d}$ transitions and from photoionization of the $2 p$ subshell (threshold at $80.2 \mathrm{eV}$ ). In the upper panel of Fig. 6, we plot the penetration depth $\delta=2 c /(\omega \kappa)$ for the case above example. In the range of experimental interest, the average-atom model predicts $\delta \approx 15 \mu \mathrm{m}$.

In Fig. 7 , we present ratios $(n-1) /\left(n_{\text {free }}-1\right)$ for an Al plasma of density $10^{20}$ ions/cc and temperatures ranging from 1 to $30 \mathrm{eV}$. For $T<4 \mathrm{eV}$, the ratio is negative for photon energies in the range of experimental interest. For 

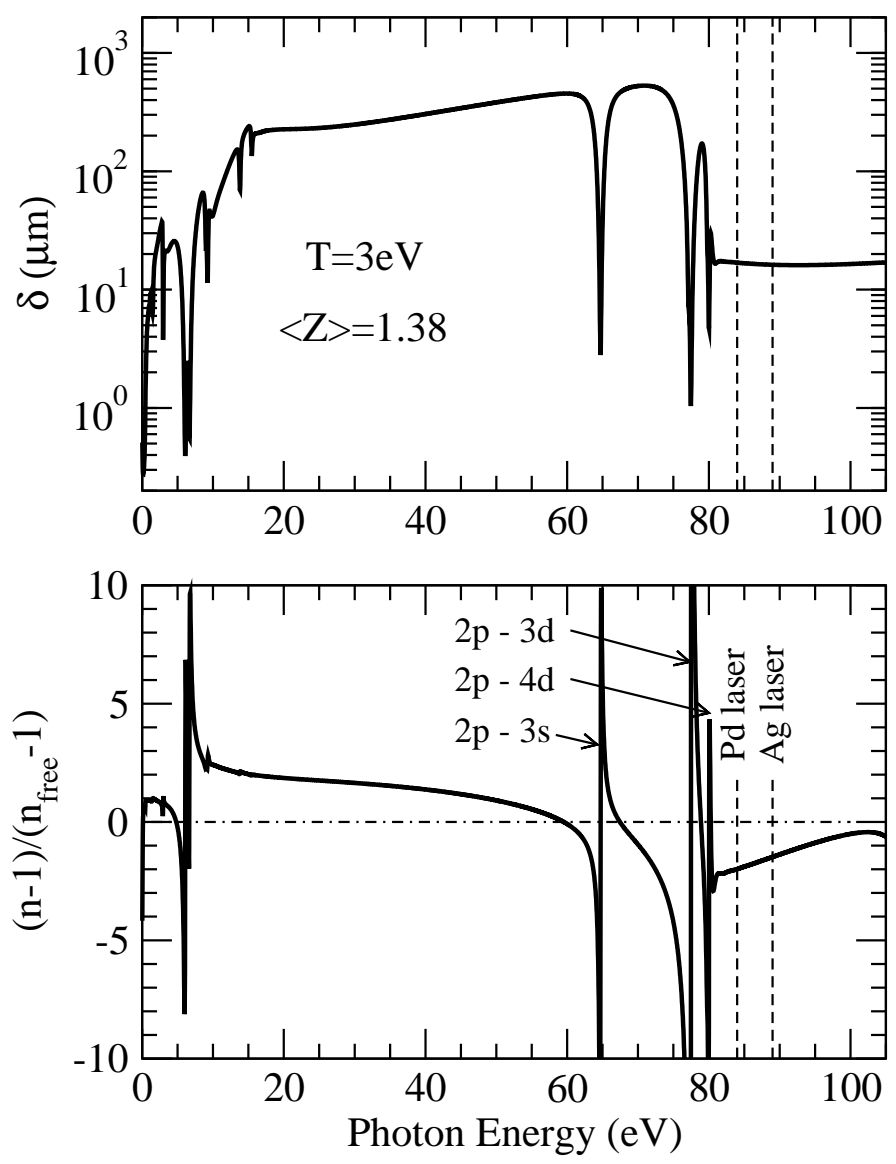

Fig. 6. Lower panel: Ratio of $(n-1)$, calculated in the average-atom model, to $\left(n_{\text {free }}-1\right)$ for an $\mathrm{Al}$ plasma at temperature $T=3 \mathrm{eV}$ and ion density $10^{20} / \mathrm{cc}$. The number of free electrons/ion is $\langle Z\rangle=1.38$. Upper panel: Penetration depth $\delta(\mu \mathrm{m})$ for the same case.

$4<T<15 \mathrm{eV}$, resonances associated with transitions from the $n=2$ shell cross the region of interest, while for $T>20 \mathrm{eV}$ the ratio is close to 1 over the entire region.

\section{Conclusion}

We have introduced an average-atom model, fashioned after the generalized Thomas-Fermi model of Feynman, Metropolis, and Teller [4], and used the model to investigate the optical properties of plasmas. Linear response of the average atom leads to a version of the Kubo-Greenwood formula for the frequency dependent conductivity $\sigma(\omega)$, which is simply related to the imaginary part of the relative dielectric function. Analytic properties of the dielectric function permit us to determine the real part of the dielectric function from its imaginary part through a dispersion integral. With the complex dielectric function in hand, we can investigate various optical properties of the plasma. 

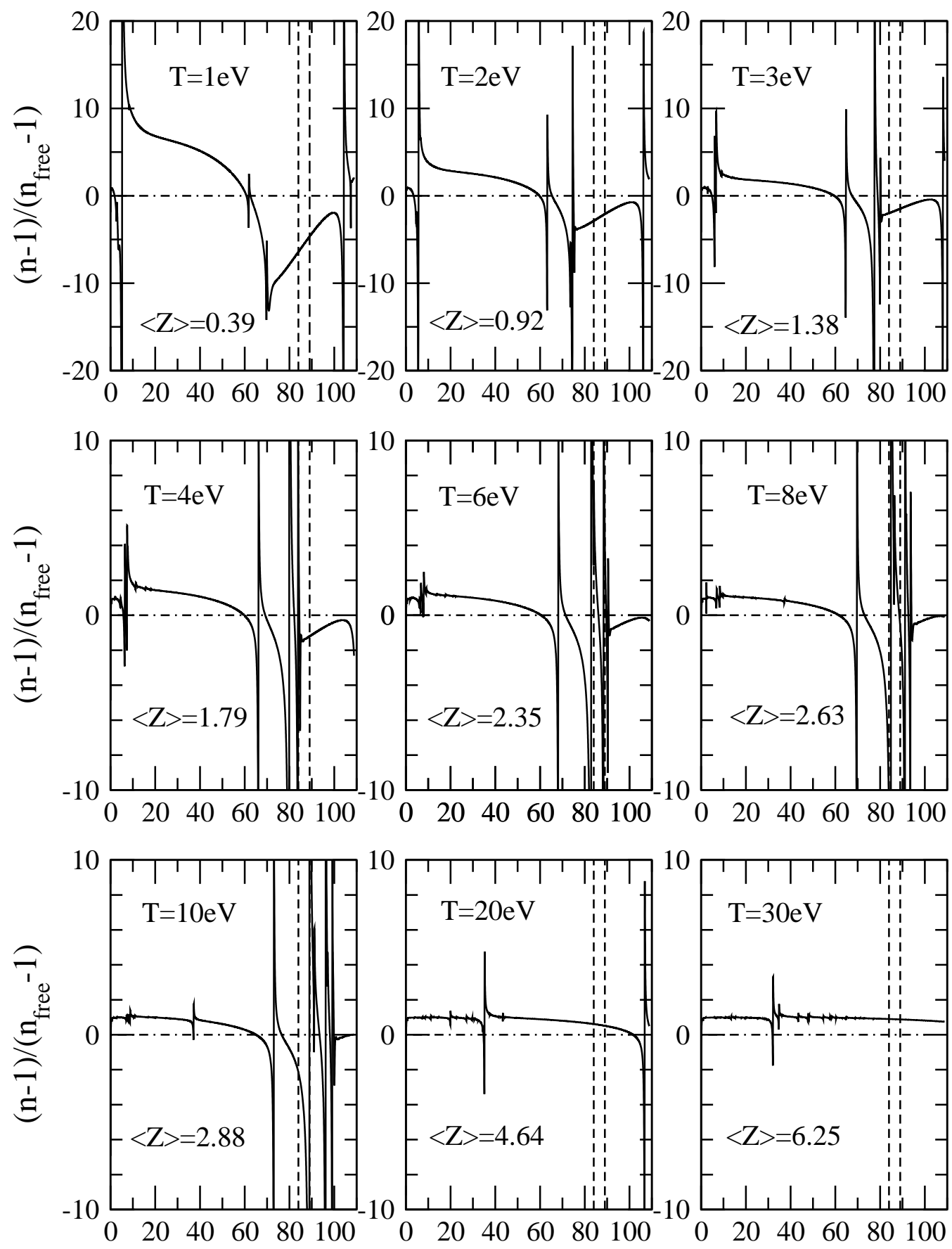

Photon Energy (eV)

Fig. 7. Ratios $(n-1) /\left(n_{\text {free }}-1\right)$ in the average-atom model for Al plasmas with temperatures in the range [1-30] eV and ion density $10^{20} / \mathrm{cc}$. Dashed vertical lines mark energies of $14.7 \mathrm{~nm}$ and $13.9 \mathrm{~nm}$ lasers.

As a specific application of the theory, we consider the index of refraction of an Al plasma, density $10^{20}$ ions/cc, in the temperature range $T=1-30 \mathrm{eV}$. At the lower end of this range, the model predicts that the index of refraction is greater than 1 , in harmony with recent observations $[1,2]$, while at the upper 
end, $n-1$ from the average-atom model agrees well with the value obtained from a free-electron analysis. Use of the free-electron model to extract electron densities from interferometric data clearly requires some preliminary study to determine whether or not bound-bound resonances and/or photoionization thresholds contribute significantly to the index of refraction. The present extension of the average-atom model provides a useful tool for investigating such questions.

\section{Acknowledgments}

The work of WRJ was supported in part by NSF grant No. PHY-01-39928 and in part by grant No. B516165 from Lawrence Livermore National Laboratory. The authors wish to thank J. Nilsen for bringing the x-ray laser interferometry experiments to their attention. Debts of thanks are also owed to J. J. Niez for explaining how to evaluate free-free matrix elements of the velocity operator in terms of Lommel integrals and to Michael Kuchiev for discussions concerning the infrared divergence of the conductivity in the average-atom model.

\section{References}

[1] J. Filevich, J. J. Rocca, M. C. Marconi, R. F. Smith, J. Dunn, R. Keenan, J. R. Hunter, S. J. Moon, J. Nilsen, J. H. Scofield, A. Ng, V. N. Shlyaptsev, Evidence of bound electron contributions to soft xray interferograms of dense plasmas, in: X-Ray Lasers 2004: Proceedings of the 9th International Conference on X-Ray Lasers, 2004.

[2] H. Tang, O. Guilbaud, G. Jamelot, D. Ros, A. Klisnik, D. Joyeux, D. Phalippou, M. Kado, M. Nishikino, M. Kishimoto, K. Sukegawa, M. Ishino, K. Nagashima, H. Daido, Diagnostics of laser-induced plasma with soft x-ray $(13.9 \mathrm{~nm}) \mathrm{bi}-$ mirror interference microscopy, Appl. Phys. B 78 (2004) 975-977.

[3] J. Nilsen, J. H. Scofield, Plasmas with index of refraction greater than one, Optics Letters 29 (2004) 2677-2679.

[4] R. P. Feynman, N. Metropolis, E. Teller, Equations of state of elements based on the generalized Fermi-Thomas theory, Phys. Rev. 75 (1949) 1561.

[5] T. Blenski, K. Ishikawa, Pressure ionization in the spherical ion-cell model of dense-plasmas and a pressure formula in the relativistic Pauli approximation, Phys. Rev. E 51 (1995) 4869-4881.

[6] R. Kubo, A general expression for the conductivity tensor, Canad. J. Phys. 34 (1956) 1274. 
[7] R. Kubo, Statistical-mechanical theory of irreversible processes. I General theory and simple applications to magnetic and conduction problems, J. Phys. Soc. Jpn. 12 (1957) 570.

[8] D. A. Greenwood, The Boltzmann equation in the theory of electrical conduction in metals, Proc. Phys. Soc. London 715 (1958) 585.

[9] W. A. Harrison, Solid State Theory, McGraw-Hill, New York, 1970.

[10] N. W. Ashcroft, N. D. Mermin, Solid-State Physics, Saunders, Philadelphia, 1976.

[11] M. P. Desjarlais, J. D. Kress, L. A. Collins, Electrical conductivity for warm, dense aluminum plasmas and liquids, Phys. Rev. E 66 (2002) 025401(R).

[12] V. Recoules, P. Renaudin, J. Cléouin, P. Noiret, G. Zérah, Electrical conductivity of hot expanded aluminum: Experimental measurements and ab initio calculations, Phys. Rev. E 66 (2002) 056412.

[13] R. de L. Kronig, On the theory of dispersion of x-rays, J. Opt. Soc. Am. 12 (1926) 547.

[14] H. A. Kramers, Some remarks of the theory of absorption and refraction of x-rays, Nature 117 (1926) 775.

[15] R. de L. Kronig, H. A. Kramers, La diffusion de la lumière par les atomes, Atti Congr. Intern. Fisici 2 (1927) 545.

[16] L. D. Landau, E. M. Lifshitz, Electrodynamics of Continuous Media, Pergamon, New York, 1984.

[17] O. Gunnarsson, B. I. Lundqvist, Exchange and correlation in atoms, molecules, and solids by spin-density functional formalism, Phys. Rev. B 13 (1976) 42744298 .

[18] J. P. Perdew, A. Zunger, Self-interaction correction to density-functional approximations for many-electron systems, Phys. Rev. B 23 (1981) 5048-5079.

[19] G. Csanak, D. P. Kilcrease, G. D. Meneses, Coupled channel formulation of the perturbed finite-temperature atomic random phase approximation: single channel approximation, J. Quant. Spectrosc. Radiat. Transfer 81 (2003) 247254 .

[20] P. K. L. Drude, Zur electronentheorie I, Annalen der Physik 1 (1900) 566-613.

[21] P. K. L. Drude, Zur electronentheorie II, Annalen der Physik 3 (1900) 369-402.

[22] G. D. Mahan, Many-Particle Physics, 3rd Edition, Plenum, 2000. 